\title{
EDITORIAL
}

\section{Evidence vs Experience in Neonatal Practices}

\section{Journal of Perinatology (2010) 30, S1; doi:10.1038/jp.2010.112}

This Supplement is the fifth proceedings of a conference titled 'Evidence vs Experience in Neonatal Practices'. This unique meeting, which had its debut in 2004, included formal lectures and discussions, facilitated by an audience response system, covering many of the most relevant topics in the care of neonates. Each speaker, nationally and internationally recognized, was given the task of addressing evolving and state-of-the-art practices based on either evidence or consensus. As program chair, I worked with Drs Ramanathan, Sekar and Seri to organize the program, which was held in Chicago, from 19 to 20 June 2009. We are pleased that once again we have had the opportunity to adapt these important presentations into the papers that appear in this Supplement.

In the United States, the rate of preterm birth is $12.8 \%$, one of the highest in the world. Preterm birth brings with it both morbidity and mortality and results in an annual cost of over $\$ 26$ billion. These are the challenges that we face in the Neonatal Intensive Care Unit (NICU) and that the Evidence vs Experience conference was created to address. Divided into four sessions, the 2009 program covered selected topics dealing with central issues in neonatology. In the first session, chaired by Dr Sekar, medical management of short gut syndrome, an update on neonatal jaundice, the changing spectrum of neonatal infections and the far-reaching effects of chorioamnionitis were discussed by Drs Bhatia, Bhutani, Plano and Kramer, respectively.

The second session, Focus on Hemodynamics and Monitoring, was chaired by Dr Ramanathan and the controversial topic of management of a patent ductus arteriosus was reviewed by $\mathrm{Dr}$ Noori. Dr Seri discussed the past, present and future of hemodynamic monitoring in neonatology, demonstrating that simply monitoring blood pressure is not enough.

Session three, chaired by Dr Seri, featured Dr Saugstad, who once again asked why we are still using oxygen to resuscitate term infants and demonstrated the short- and long-term morbidities associated with the use of supplemental oxygen during resuscitation in term infants. This session ended with Dr Sekar discussing the continuing problems of iatrogenic complications in the NICU.

The last session featured our key note speaker, Dr Finer, introduced by Dr Ramanathan, who discussed the 'golden hour' of newborn resuscitation. The session continued with the use of noninvasive nasal ventilation as a primary mode after surfactant replacement in premature infants (Dr Ramanathan), the use of amplitude-integrated electroencephalography in the neurological assessment of term and preterm infants (Dr Mathur), and ended with a review of evidence regarding hypothermia in the treatment of hypoxic ischemic encephalopathy by Dr Soll.

I wish to recognize the many contributions of the steering committee, who also served as guest editors for this publication. I extend my sincere appreciation to our renowned faculty who took the time to share their expertise, participate in the discussions and develop these papers. I also wish to thank the Annenberg Center for Health Sciences for organizing and running the conference, BioComm Network for assistance in compiling the manuscripts, and, of course, our partners at the Nature Publishing Group. This conference would not have been possible without the support of Dey, LP - their educational support over the years allowed this sixth conference to grow to over 300 participants. This Supplement, in turn, would not have been published without a grant from Cornerstone Therapeutics Inc., for which we are very grateful. Lastly, I must thank and commend all the participants who shared in the success of this conference. I look forward to everyone joining us next year.

J Bhatia

Medical College of Georgia, Augusta, GA, USA E-mail: jatindeb@mcg.edu 